\title{
Energy diffusion and absorption in chaotic systems with rapid periodic driving
}

\author{
Wade Hodson $\odot^{*}$ \\ Department of Physics, University of Maryland, College Park, Maryland 20742, USA \\ Christopher Jarzynski $\oplus^{\dagger}$ \\ Institute for Physical Science and Technology, Department of Chemistry and Biochemistry, \\ and Department of Physics, University of Maryland, College Park, Maryland 20742, USA
}

(Received 31 July 2020; accepted 25 February 2021; published 8 March 2021)

\begin{abstract}
When a chaotic, ergodic Hamiltonian system with $N$ degrees of freedom is subject to sufficiently rapid periodic driving, its energy evolves diffusively. We derive a Fokker-Planck equation that governs the evolution of the system's probability distribution in energy space, and we provide explicit expressions for the energy drift and diffusion rates. Our analysis suggests that the system generically relaxes to a long-lived prethermal state characterized by minimal energy absorption, eventually followed by more rapid heating. When $N \gg 1$, the system ultimately absorbs energy indefinitely from the drive, or at least until an infinite temperature state is reached.
\end{abstract}

DOI: 10.1103/PhysRevResearch.3.013219

\section{INTRODUCTION}

Time-periodic driving facilitates a rich range of classical and quantum dynamical behaviors, including synchronization and resonance [1-4], localization [5-8], and chaos [1,9]. Recent theoretical and experimental work has aimed to identify nonequilibrium phases of matter that might emerge in periodically driven systems [10-12]. Phenomena such as time crystallization [11-17] and prethermalization [12,17-28] reveal that periodic driving can stabilize systems in a variety of interesting and useful states.

Energy absorption poses a potential obstacle to such stabilization of nonequilibrium states of matter. A driven open system in a nonequilibrium steady state attains a balance in which energy absorbed from the drive is dissipated into an environment, such as a thermal bath. But if a system is isolated, save its interaction with the drive, then maintaining a stable state requires the suppression of energy absorption from the drive. Much work has been devoted to understanding energy absorption and the conditions under which it might be suppressed, in periodically driven, isolated classical and quantum systems [18,20,23-25,29-39].

In this paper, we study the general problem of energy absorption in isolated classical chaotic systems subject to rapid periodic driving. We argue that the energetic dynamics of such systems are diffusive, and we derive a Fokker-Planck equation for the evolution of the system's energy probability distribution, $\eta(E, t)$. The drift and diffusion coefficients in this

\footnotetext{
*whodson@umd.edu

†cjarzyns@umd.edu
}

Published by the American Physical Society under the terms of the Creative Commons Attribution 4.0 International license. Further distribution of this work must maintain attribution to the author(s) and the published article's title, journal citation, and DOI. equation, characterizing energy absorption and the spreading of the energy distribution, are given explicitly in terms of the dynamics of the undriven system - much as in the case of ordinary linear response theory (LRT) [40] but without the assumption of weak driving. For many-body systems, our results suggest a scenario marked by three stages: initial relaxation to an equilibriumlike prethermal state [12,18-28], followed by a long interval of minimal energy absorption, and finally rapid absorption toward an infinite-temperature state.

Our description provides a comprehensive, quantitative account of energy absorption in rapidly and periodically driven chaotic systems. It reveals how chaos in phase space facilitates stochastic energy evolution, how energy diffusion leads to the breakdown of the prethermal regime, and how energy absorption rates are determined by the underlying, undriven Hamiltonian dynamics. Our framework also suggests a generic explanation for the exponential-in-frequency suppression of energy absorption observed in a range of systems $[12,18,20,23,24,27,28,31,35,36,39]$. Finally, we argue that the classical results that we obtain are relevant to energy absorption in quantum systems, in an appropriate semiclassical limit.

In Sec. II, we define the problem we will study. In Sec. III, we argue that the energy of a rapidly driven chaotic system evolves diffusively, and we derive the Fokker-Planck equation that describes this evolution. In Sec. IV, we analyze energy absorption and prethermalization in the context of our energy diffusion model. In Sec. V, we briefly consider the quantum counterpart of our classical problem, and we conclude in Sec. VI.

\section{SETUP}

Our object of study is a classical Hamiltonian system with $N \geqslant 2$ degrees of freedom. At time $t$, the microscopic state of the system is specified by a phase space point $z_{t} \equiv z \equiv(\mathbf{q}, \mathbf{p})$, where the $N$-vectors $\mathbf{q}$ and $\mathbf{p}$ specify canonical coordinates 
and momenta. The system evolves under Hamilton's equations of motion, generated by a Hamiltonian $H \equiv H(z, t)$ :

$$
\frac{d \mathbf{q}}{d t}=\frac{\partial H}{\partial \mathbf{p}}, \quad \frac{d \mathbf{p}}{d t}=-\frac{\partial H}{\partial \mathbf{q}} .
$$

For an ensemble of trajectories, the phase space probability distribution $\rho(z, t)$ obeys the Liouville equation,

$$
\frac{\partial \rho}{\partial t}=\{H, \rho\}=\frac{\partial H}{\partial \mathbf{q}} \cdot \frac{\partial \rho}{\partial \mathbf{p}}-\frac{\partial H}{\partial \mathbf{p}} \cdot \frac{\partial \rho}{\partial \mathbf{q}},
$$

where $\{\cdot, \cdot\}$ denotes the Poisson bracket [41]. We take $H(z, t)$ to be a periodic function of time, with period $T$, and we decompose this Hamiltonian into its time average $H_{0}(z) \equiv$ $T^{-1} \int_{0}^{T} d t H(z, t)$, and a remainder $V(z, t)=V(z, t+T)$ with vanishing average:

$$
H(z, t)=H_{0}(z)+V(z, t) .
$$

We will refer to $H_{0}(z)$ as the bare or undriven Hamiltonian, and to $V(z, t)$ as the drive. The former determines the evolution of the system in complete isolation, that is, when $V=0$. We define the system's energy $E(t)$ to be the bare Hamiltonian evaluated at $z_{t}: E(t) \equiv H_{0}\left(z_{t}\right)$. When $V=0$, the energy is a constant of the motion: any trajectory $z_{t}$ is constrained to evolve on an energy shell, that is, a level surface of $H_{0}(z)$. The evolution of the energy when $V \neq 0$ will be our central focus. The magnitude of the drive $V(z, t)$ is arbitrary; in particular, we do not assume it to be small.

We assume that the undriven dynamics are chaotic and ergodic on each energy shell of $H_{0}$. Such dynamics exhibit mixing as trajectories diverge from one another exponentially with time [9]. This leads to a loss of statistical dependence between states of the system at different times, as reflected in the decay of correlations-in effect, the system loses its memory of previously visited states. After a characteristic mixing time, any smooth initial distribution on the energy shell evolves to a distribution that for practical purposes is microcanonical, or thermal $[40,42]$. Thus chaos offers a way to understand the self-thermalizing properties of many-body systems such as gases and liquids, while also providing low-dimensional analogs, such as chaotic billiard systems, that are accessible to numerical or analytical study.

We are interested in the limit of high driving frequency $\omega=2 \pi / T$. When $\omega \rightarrow \infty$, the effect of the drive averages to zero over each period, as the system cannot appreciably react to the drive in such a short time. In this limit, the evolution generated by the driven Hamiltonian approaches the undriven evolution: For given initial conditions $z_{0}$, and over a fixed time interval $0 \leqslant t \leqslant \tau$, the trajectory $z_{t}$ that evolves under $H(z, t)$ converges, as $\omega \rightarrow \infty$, to the trajectory $z_{t}^{0}$ that evolves under $H_{0}(z)[43,44]$. This limit can be attained regardless of the strength of the drive, $V(z, t)$. In our case, we assume $\omega$ is sufficiently high such that driven and undriven trajectories remain close over timescales characteristic of the decay of correlations.

These considerations lead to the following picture. For sufficiently short times, energy is approximately conserved and the driven trajectories $z_{t}$ generated by $H(z, t)$ are similar to the undriven trajectories $z_{t}^{0}$ generated by $H_{0}(z)$, allowing us to use the latter to estimate correlation functions that will arise in our analysis. These correlation functions, as we shall see, will in turn describe how the system absorbs energy from the external drive on much longer timescales.

\section{ENERGY DIFFUSION}

Given the assumptions mentioned in Sec. II, we now argue that the energy of the driven system evolves diffusively. For simplicity, we consider monochromatic driving:

$$
V(z, t)=V(z) \cos (\omega t) .
$$

Our analysis can be generalized to arbitrary time-periodic driving by decomposing $V(z, t)$ in a Fourier series with fundamental frequency $\omega$.

\section{A. Argument for energy diffusion}

To begin, we consider a system that evolves over a time interval $0 \leqslant t \leqslant \Delta t$, from initial conditions $z_{0}$ sampled from a microcanonical distribution at energy $E_{0}$ :

$$
\rho\left(z_{0}, 0\right)=\rho_{E_{0}}\left(z_{0}\right) \equiv \frac{1}{\Sigma\left(E_{0}\right)} \delta\left(E_{0}-H_{0}\left(z_{0}\right)\right) .
$$

Here

$$
\Sigma(E)=\frac{\partial \Omega}{\partial E}=\int d z \delta\left(H_{0}(z)-E\right)
$$

is the classical density of states,

$$
\Omega(E)=\int d z \theta\left(E-H_{0}(z)\right)
$$

is the phase space volume enclosed by the energy shell $E$, which we assume to be finite for all $E$, and the integrals are over phase space. Let $\Delta E\left(z_{0}\right)$ denote the net change in the system's energy from $t=0$ to $t=\Delta t$. By Hamilton's equations, $\Delta E\left(z_{0}\right)$ is the time integral of the power

$$
\frac{d E}{d t} \equiv \frac{d}{d t} H_{0}\left(z_{t}\right)=-\cos (\omega t) \dot{V}\left(z_{t}\right),
$$

where $\dot{V}(z) \equiv\left\{V, H_{0}\right\}$. The quantity $\Delta E$ can be viewed as a random variable, whose value is determined by the sampled initial conditions $z_{0}$. Understanding the statistics of $\Delta E$ in the high-frequency driving regime, for an appropriate choice of $\Delta t$ (to be clarified below), will be the key to establishing diffusion in energy space.

[We note in passing that if the undriven Hamiltonian has the form $H_{0}=\sum_{n}\left(\mathbf{p}_{n}^{2} / 2 m_{n}\right)+U_{0}\left(\left\{\mathbf{q}_{n}\right\}\right)$ and if the drive $V$ depends on coordinates $\left\{\mathbf{q}_{n}\right\}$ but not momenta $\left\{\mathbf{p}_{n}\right\}$, then Eq. (8) becomes $d E / d t=\sum_{n} \mathbf{F}_{n} \cdot \mathbf{v}_{n}$, where $\mathbf{F}_{n}$ is the driving force acting on the $n$th particle, and $\mathbf{v}_{n}$ is that particle's velocity.]

We now explicitly assume the driving is rapid. To begin, we impose the condition

$$
T \ll \tau_{C}\left(E_{0}\right),
$$

where $T=2 \pi / \omega$ is the drive period and $\tau_{C}\left(E_{0}\right)$ is a characteristic timescale over which chaotic mixing on the energy shell $E_{0}$ produces the decay of correlations. Heuristically, Eq. (9) implies that a trajectory $z_{t}$ travels only a negligible distance during one period of driving. This condition produces the averaging over oscillations that (as mentioned in Sec. II) results in driven trajectories $z_{t}$ resembling their undriven counterparts $z_{t}^{0}$. Let us now choose $\Delta t$ so that over the interval $[0, \Delta t]$ the 
driven trajectories in our ensemble remain close to the initial energy shell $E_{0}$. Chaotic mixing ensures then that a microcanonical distribution is approximately maintained. Thus for any $t \in[0, \Delta t]$, the ensemble of points $z_{t}$ are approximately distributed according to the initial microcanonical ensemble.

With this picture in mind, let us divide the time interval $[0, \Delta t]$ into $M \gg 1$ subintervals of equal duration $\delta t=$ $\Delta t / M$, and consider $\Delta E=\sum_{i} \delta E_{i}$ as a sum of subinterval energy changes $\delta E_{i}, i=1,2 \cdots M$. Each increment $\delta E_{i}$ is itself a random variable, determined by integrating the power Eq. (8) along the trajectory $z_{t}$ over the subinterval. By the arguments of the previous paragraph, the $\delta E_{i}$ 's have nearly identical, microcanonical statistics, provided we choose $\delta t$ (and therefore also $\Delta t$ ) to be an integer multiple of the driving period $T$ to ensure that each subinterval begins at the same phase of the drive.

Chaotic mixing on the energy shell $E_{0}$ produces the decay of correlations. Let us further choose $\delta t$ to be longer than the characteristic correlation time $\tau_{C}\left(E_{0}\right)$, so each $\delta E_{i}$ is approximately statistically independent from the others. The energy change $\Delta E$ is then a sum of $M \gg 1$ approximately independent and identically distributed increments $\delta E_{i}$ : the system effectively performs a random walk on the energy axis. By the central limit theorem, $\Delta E$ is a normally distributed random variable, whose mean and variance grow (for fixed $\delta t$ ) in proportion to the number of increments $M$, equivalently the time elapsed $\Delta t$.

The statistical behavior just described is characteristic of a diffusive process in energy space, motivating us to model it by a Fokker-Planck equation [45]. Letting

$$
\eta(E, t) \equiv \int d z \delta\left(H_{0}(z)-E\right) \rho(z, t)
$$

denote the energy distribution, we postulate that the time evolution of $\eta$ is given by

$$
\frac{\partial \eta}{\partial t}=-\frac{\partial}{\partial E}\left(g_{1} \eta\right)+\frac{1}{2} \frac{\partial^{2}}{\partial E^{2}}\left(g_{2} \eta\right) .
$$

The drift and diffusion coefficients $g_{1}(E, \omega)$ and $g_{2}(E, \omega)$ characterize, respectively, the rate at which the distribution $\eta$ shifts and spreads on the energy axis; see Eqs. (13) and (14) below. These coefficients depend on the system energy $E$ and the driving frequency $\omega$. Energy diffusion and its description in terms of the Fokker-Planck equation have been studied in various contexts involving externally driven Hamiltonian systems [32,46-56]. Before deriving expressions for $g_{1}$ and $g_{2}$ in the high-frequency driving regime, it is worth examining the central role that a separation of timescales plays in our analysis.

We have assumed, after Eq. (9), that $\Delta t$ is much smaller than the timescale $\tau_{E}\left(\omega, E_{0}\right)$ over which the energy of the system changes significantly. This condition ensures that the energy increments $\delta E_{i}$ have approximately identical microcanonical statistics. We have also assumed that the interval $\Delta t$ contains many subintervals of duration $\delta t$, and that $\delta t>$ $\tau_{C}\left(E_{0}\right)$, guaranteeing approximate statistical independence among the increments $\delta E_{i}$. Thus, our analysis involves the hierarchy of timescales:

$$
T \ll \tau_{C}\left(E_{0}\right) \ll \Delta t \ll \tau_{E}\left(\omega, E_{0}\right) .
$$

Since $\tau_{E} \rightarrow \infty$ as $\omega \rightarrow \infty$, this hierarchy can be satisfied for any particular energy shell $E_{0}$ by setting $\omega$ sufficiently large. We conclude that Eq. (11) is valid over an interval of the energy axis whose extent is determined by, and increases with, the value of $\omega$.

The above arguments suggest that the energy diffusion description is valid on a coarse-grained timescale of order $\Delta t$. On shorter timescales, computing the fine details of the system's energy evolution requires the full Hamiltonian equations of motion Eq. (1). These details vary greatly from system to system. However, as we will see, the characteristics of the energy diffusion process ultimately depend only on a few key details of these system-specific dynamics, as captured in the coefficients $g_{1}$ and $g_{2}$.

\section{B. Drift and diffusion coefficients}

Under Eq. (11), an initial distribution $\eta(E, 0)=\delta\left(E-E_{0}\right)$ evolves after a time $\Delta t \ll \tau_{E}$ to a distribution $\eta(E, \Delta t)$ with mean and variance [45]:

$$
\begin{gathered}
\operatorname{Mean}(E)=E_{0}+g_{1}\left(E_{0}, \omega\right) \Delta t, \\
\operatorname{Var}(E)=g_{2}\left(E_{0}, \omega\right) \Delta t .
\end{gathered}
$$

We can thus determine $g_{2}$ by calculating $\operatorname{Var}(E)$, the energy spread acquired by an ensemble of trajectories with initial energy $E_{0}$, evolved for a time $\Delta t$ under the driven Hamiltonian. We perform this calculation in Sec. A 1 of the Appendix, obtaining, in the limit of large $\omega$,

$$
\begin{gathered}
g_{2}(E, \omega)=\frac{1}{2} S(\omega ; E) \geqslant 0, \\
S(\omega ; E)=\int_{-\infty}^{\infty} d t e^{-i \omega t} C(t ; E),
\end{gathered}
$$

where

$$
C(t ; E) \equiv\left\langle\dot{V}\left(z_{0}^{0}\right) \dot{V}\left(z_{t}^{0}\right)\right\rangle-\left\langle\dot{V}\left(z_{0}^{0}\right)\right\rangle\left\langle\dot{V}\left(z_{t}^{0}\right)\right\rangle
$$

is the microcanonical autocorrelation function of the observable $\dot{V}(z)$. Specifically, the averages denoted by $\langle\cdot\rangle$ are computed by sampling initial conditions $z_{0}^{0}$ from a microcanonical ensemble at energy $E$, then evolving for time $t$ under $H_{0}(z)$. By the Wiener-Khinchin theorem [57], the Fourier transform of $C(t ; E)$ is the power spectrum of $\dot{V}\left(z_{t}^{0}\right)$ at energy $E$, denoted by $S(\omega ; E)$. Note that Eq. (15) gives $g_{2}$ entirely in terms of properties of the undriven system, as $C(t ; E)$ and thus $S(\omega ; E)$ are defined in terms of the undriven trajectories $z_{t}^{0}$.

In solving for $g_{2}$, we approximated driven trajectories $z_{t}$ by their undriven counterparts $z_{t}^{0}$. As a result, we expect that Eq. (15) contains correction terms that become negligible in the high-frequency limit $\omega \rightarrow \infty$.

In Sec. A 2 of the Appendix, we use Liouville's theorem, which expresses the incompressibility of phase space volume under Hamiltonian dynamics [40] to obtain the following expression for the drift coefficient $g_{1}$ in terms of $g_{2}(E, \omega)$ and the density of states $\Sigma(E)$ Eq. (6):

$$
g_{1}(E, \omega)=\frac{1}{2 \Sigma} \frac{\partial}{\partial E}\left(g_{2} \Sigma\right) .
$$


This result is a fluctuation-dissipation relation, similar to others previously established for various driven Hamiltonian systems [47-49,51-54].

Using Eqs. (15) and (18), the Fokker-Planck Eq. (11) takes the compact form

$$
\frac{\partial \eta}{\partial t}=\frac{1}{4} \frac{\partial}{\partial E}\left[S \Sigma \frac{\partial}{\partial E}\left(\frac{\eta}{\Sigma}\right)\right] .
$$

Equation (19) is our main result. It describes the stochastic evolution of the system's energy, under rapid driving, in terms of quantities $S(\omega ; E)$ and $\Sigma(E)$ that characterize the undriven system.

As discussed earlier, we expect Eq. (19) to be valid over a region of the energy axis whose extent depends on $\omega$. In the next section, we assume $\omega$ is sufficiently large that Eq. (19) is valid over the entire energy axis [58].

\section{ENERGY ABSORPTION AND PRETHERMALIZATION}

\section{A. Energy absorption}

We now consider energy absorption, focusing on manybody systems. Under what conditions does the system absorb energy from the rapid drive? Multiplying the Fokker-Planck Eq. (11) by $E$ and integrating over energy, we obtain

$$
\frac{d\langle E\rangle}{d t}=\left\langle g_{1}(E, \omega)\right\rangle,
$$

where $\langle f\rangle \equiv \int d E \eta f$ for any $f(E)$. Defining a microcanonical temperature $T_{\mu}(E)$ via

$$
\frac{1}{T_{\mu}}=\frac{\partial s}{\partial E},
$$

where $s(E) \equiv k_{B} \ln \Sigma(E)$ is the microcanonical entropy and $k_{B}$ is Boltzmann's constant, Eq. (18) becomes

$$
g_{1}(E, \omega)=\frac{1}{2 k_{B} T_{\mu}}\left[g_{2}(E, \omega)+k_{B} T_{\mu} \frac{\partial g_{2}(E, \omega)}{\partial E}\right] .
$$

The expression in square brackets is an expansion of $g_{2}(E+$ $\left.k_{B} T_{\mu}, \omega\right)$ for small $k_{B} T_{\mu}$, truncated after first order. For a system with $N$ degrees of freedom, the difference between $E$ and $E+k_{B} T_{\mu}$ corresponds to an energy change of $k_{B} T_{\mu} / N$ per degree of freedom. When $N \gg 1$ this change is negligible and Eqs. (15), (20), (22) give

$$
\frac{d\langle E\rangle}{d t}=\left\langle\frac{S(\omega ; E)}{4 k_{B} T_{\mu}}\right\rangle .
$$

For a many-body system with an unbounded phase space, such as a gas or liquid, the density of states $\Sigma(E)$ increases with energy, hence $T_{\mu}(E)>0$ and Eqs. (15) and (23) imply that the average energy of the system continually increases with time, as expected intuitively.

If the phase space of the system is bounded, then we expect $T_{\mu}(E)<0$ at some energies. For example, for $N$ classical spins described by $H_{0}=\mathbf{B} \cdot \sum_{n} \mathbf{S}_{n}, T_{\mu}(E)<0$ when $E>0$. Thus $d\langle E\rangle / d t$ can be negative. In this situation, we can view the normalized density of states, $\bar{\Sigma}(E) \equiv \Sigma(E) / \int d E^{\prime} \Sigma\left(E^{\prime}\right)$, as the infinite temperature energy distribution, obtained by considering the canonical energy distribution $\eta_{T_{c}}(E) \propto$ $\Sigma(E) e^{-E / k_{B} T_{c}}$ in the limit $T_{c} \rightarrow \infty$. If $g_{2}(E)$ is strictly positive for all $E$, ensuring that there are no insurmountable barriers along the energy axis, then Eq. (19) describes an ergodic Markov process, and $\bar{\Sigma}(E)$ is the unique stationary distribution to which any initial distribution evolves as $t \rightarrow \infty$ $[59,60]$.

We thus identify two possible energetic fates of a manybody system in the rapid driving regime. If the phase space is unbounded, then the average energy of the system increases indefinitely whereas if the system admits a normalized stationary distribution $\bar{\Sigma}(E)$, then the system evolves to this infinite temperature distribution.

\section{B. Prethermalization}

In either case, the energy dynamics predicted by the energy diffusion description relate to the phenomenon of prethermalization. A driven system is said to prethermalize if it reaches thermal equilibrium with respect to an effective Hamiltonian on short to intermediate timescales before ultimately gaining energy at far longer times [12,18-28]. In our case, if the system is prepared in a nonmicrocanonical (i.e., nonequilibrium) distribution on a particular energy shell $E_{0}$, then after a characteristic mixing time the distribution on this energy shell becomes effectively microcanonical, i.e., prethermalization occurs with respect to $H_{0}$, at nearly constant energy. On longer timescales, the energy dynamics are governed by the Fokker-Planck Eq. (19), and the system absorbs energy from the drive $V(t)$ Eq. (23). For large $\omega$, this absorption can be exceedingly slow, as the power spectrum $S(\omega ; E)$ decays faster than any power of $\omega^{-1}$ for any smooth $H_{0}$ [61]. This is consistent with observed exponential-in-frequency suppression of energy absorption in a range of classical and quantum model systems [12,18,20,23,24,27,28,31,35,36,39]. Prethermalization thus occurs when $\omega$ lies deep within the tail of the power spectrum.

Following the above-mentioned initial relaxation, energy absorption is slow but does not vanish. As the system energy $E$ gradually grows, the intrinsic correlation time $\tau_{C}(E)$ generically decreases with increasing particle velocities, hence the power spectrum $S(\omega ; E)$ broadens. Eventually, at sufficiently large $E$, the drive frequency might no longer be located in the far tail of the power spectrum: This marks the onset of unsuppressed energy absorption toward the infinite-temperature state. If the phase space is bounded, then $\omega$ can be chosen so that energy absorption is suppressed on all energy shells; in this case, energy absorption remains very slow throughout the system's evolution toward the infinite-temperature energy distribution $\bar{\Sigma}(E)$.

Energy absorption from periodic driving has also been studied using the Floquet-Magnus (FM) expansion, which, for time-periodic $H(z, t)$, expresses the associated Floquet Hamiltonian $H_{F}(z)$ as a perturbative expansion in powers of $\omega^{-1} . H_{F}$ is a time-independent Hamiltonian whose dynamics coincide with those of $H(t)$ at stroboscopic times $t=0, T, 2 T \ldots$. At high frequencies and short timescales, the evolution obtained by truncating the FM expansion at some order is expected to be a good approximation of the exact dynamics [18,44,62-64]. See, e.g., the fourth-order (in $\omega^{-1}$ ) expression for $H_{F}(z)$ derived in Refs. [44,62] for a system in one degree of freedom. By contrast it appears that our results 
are not obtainable via the FM expansion. For smooth $H_{0}$, the coefficient $g_{2}(E, \omega)$ decays faster than any power of $\omega^{-1}$ at large $\omega$ (as mentioned earlier), and thus cannot be described accurately by an FM-like expansion in powers of $\omega^{-1}$. Indeed, this might have been anticipated, as high-frequency driving cannot induce unbounded energy absorption unless the FM expansion diverges $[18,21,33,63]$.

\section{QUANTUM-CLASSICAL CORRESPONDENCE}

Energy absorption, prethermalization, and relaxation to the infinite temperature state have been documented for a variety of periodically driven quantum systems [18,29,30,33,34,54]. It is instructive to ask how the classical energy diffusion described by Eq. (19) might emerge, in agreement with the correspondence principle, as the semiclassical limit of quantum dynamics. We now briefly describe a model that illustrates this correspondence; similar analyses may be found elsewhere in the literature on energy diffusion [53,65].

Consider a quantum system governed by a Hamiltonian $\hat{H}_{0}+\hat{V} \cos (\omega t)$, the counterpart of Eq. (3). Let us model the system's evolution as a random walk in the spectrum of $\hat{H}_{0}$, with stochastic quantum jumps from one energy level to another. By Fermi's golden rule, the transition rate from energy $E$ to $E \pm \hbar \omega$ is given by

$$
\Gamma_{ \pm}=\frac{\pi}{2 \hbar} \overline{\left|V_{m n}\right|^{2}} \rho\left(E_{n}\right),
$$

where $V_{m n}=\langle m|\hat{V}| n\rangle$ is the matrix element of $\hat{V}$ associated with the energy levels $E_{m}$ and $E_{n}$ of $\hat{H}_{0}$; the overbar denotes an average over a narrow range of matrix elements with $E_{m} \approx E$ and $E_{n} \approx E \pm \hbar \omega$; and $\rho(E)=\Sigma(E) / h^{N}$ is the semiclassical density of states. As $\hbar \rightarrow 0$, the spectrum of $\hat{H}_{0}$ becomes dense and our random walk model leads naturally to a description in terms of energy diffusion, with drift and diffusion coefficients:

$$
g_{1}=\left(\Gamma_{+}-\Gamma_{-}\right)(\hbar \omega), \quad g_{2}=\left(\Gamma_{+}+\Gamma_{-}\right)(\hbar \omega)^{2} .
$$

A semiclassical estimate for matrix elements of quantized chaotic systems [66,67] gives

$$
\overline{\left|V_{m n}\right|^{2}} \approx h^{N-1} \frac{S_{V}(\omega ; \bar{E})}{\Sigma(\bar{E})},
$$

where $\bar{E} \equiv\left(E_{m}+E_{n}\right) / 2$. Here, $S_{V}(\omega ; E)$ is the power spectrum for the classical observable $V$ and is related to $S(\omega ; E)$ (the power spectrum for $\dot{V}$ ) via $S=\omega^{2} S_{V}$. Combining results, we find that Eq. (25) converges to the classical results Eqs. (18) and (15) as $\hbar \rightarrow 0$. While this analysis is based on a heuristic model that ignores quantum coherences, it suggests that our classical energy diffusion picture is relevant for understanding periodically driven quantum systems; in particular, it provides a semiclassical explanation for the observed exponential-in-frequency suppression of energy absorption [12,18,24,27,28,31,35,36,39].

\section{CONCLUSION}

We have analyzed the diffusive energy dynamics of chaotic, ergodic Hamiltonian systems under rapid periodic driving. Observing that the system's dynamics are only weakly affected by very rapid driving, we have established a Fokker-Planck equation governing the evolution of the system's energy probability distribution. Our analysis predicts a generic, long-lived prethermal state, and for manybody systems our results point to two possible energetic fates: indefinite energy growth or relaxation to the infinitetemperature equilibrium state. In the semiclassical limit, a model of energy absorption for periodically driven, quantized chaotic systems coincides with our purely classical energy diffusion description.

A central feature of our Fokker-Planck equation is that the drift and diffusion coefficients $g_{1}$ and $g_{2}$ are determined by the undriven dynamics. A similar situation arises in LRT, where transport coefficients, such as electrical conductivities, in a system subject to weak time-periodic driving, are expressed in terms of correlation functions computed in the absence of driving [40]. In LRT, these results are obtained perturbatively through a formal expansion in powers of the driving strength. It is unclear whether our results can similarly be obtained through a perturbative expansion. A natural candidate for a small parameter in our case is the inverse frequency $\omega^{-1}$, but this seems to lead to the FM expansion, which as already noted at the end of Sec. IV B is somewhat at odds with our analysis. Both this discrepancy, and the question of whether our results can be obtained through a formal perturbative expansion, bear further investigation.

Low-dimensional billiard systems - in which a particle in a cavity alternates between straight-line motion and specular reflection off the cavity walls - offer an ideal testing ground for the theory presented in this paper, as certain billiard shapes are rigorously proven [68-70] to generate chaotic, ergodic motion. Energy absorption in driven billiard systems, sometimes known as Fermi acceleration, is a well-studied phenomenon [32,52,71-74], although much of the existing literature focuses on the case of slow driving. In a forthcoming work, we will present numerical evidence for the validity of the Fokker-Planck Eq. (19) for a particle in a chaotic billiard subject to a spatially uniform, rapidly time-periodic force. For this system, the driven and undriven trajectories of the particle can be computed to machine precision and Eq. (19) can be solved analytically, allowing for an especially precise test of the energy diffusion description.

Our results may also be tested for previously studied manybody classical systems, such as a many-body generalization of the kicked rotor model [9,75] that exhibits unbounded energy absorption in a range of parameter regimes [25,37,38]. Energy absorption has also been studied in the classical driven Heisenberg spin chain [4,20,23]. For these models and others, we expect our analysis to apply only if the time-averaged Hamiltonian $H_{0}$ generates chaotic and ergodic dynamics.

\section{ACKNOWLEDGMENTS}

We gratefully acknowledge stimulating discussions with Ed Ott, David Levermore, and Saar Rahav, and financial support from the DARPA DRINQS program (D18AC00033).

\section{APPENDIX}

Here, we obtain an expression for the energy diffusion coefficient $g_{2}$, given by Eq. (15). We then derive the fluctuation-dissipation relation Eq. (18). 


\section{Calculation of $g_{2}$}

We begin with relation Eq. (14). According to this equation, calculating $g_{2}$ amounts to computing $\operatorname{Var}(E)$, the variance in energy acquired by an ensemble of trajectories with initial energy $E_{0}$, evolved for a time $\Delta t$ under the driven Hamiltonian. Specifically, we consider an ensemble of driven trajectories evolving from microcanonically sampled initial conditions at $t=0$. Upon integrating Eq. (8) along these trajectories, we obtain (with no approximations so far)

$$
\operatorname{Var}(E)=\int_{0}^{\Delta t} \int_{0}^{\Delta t} d t d t^{\prime} \cos (\omega t) \cos \left(\omega t^{\prime}\right) C_{\mathrm{neq}}\left(t, t^{\prime} ; E_{0}\right)
$$

where $C_{\text {neq }}\left(t, t^{\prime} ; E_{0}\right) \equiv\left\langle\dot{V}\left(z_{t}\right) \dot{V}\left(z_{t^{\prime}}\right)\right\rangle-\left\langle\dot{V}\left(z_{t}\right)\right\rangle\left\langle\dot{V}\left(z_{t^{\prime}}\right)\right\rangle$ is a nonequilibrium correlation function and angular brackets $\langle\cdot\rangle$ denote an ensemble average. In the high-frequency limit $\omega \rightarrow$ $\infty$, as driven trajectories $z_{t}$ approach their undriven counterparts $z_{t}^{0}, C_{\text {neq }}\left(t, t^{\prime} ; E_{0}\right)$ can be replaced by the equilibrium correlation function

$$
C\left(t^{\prime}-t ; E_{0}\right) \equiv\left\langle\dot{V}\left(z_{t}^{0}\right) \dot{V}\left(z_{t^{\prime}}^{0}\right)\right\rangle-\left\langle\dot{V}\left(z_{t}^{0}\right)\right\rangle\left\langle\dot{V}\left(z_{t^{\prime}}^{0}\right)\right\rangle,
$$

which depends only on the difference $t^{\prime}-t$, due to the timetranslation symmetry of the microcanonical distribution under the undriven dynamics.

Replacing $C_{\text {neq }}\left(t, t^{\prime} ; E_{0}\right)$ by $C\left(t^{\prime}-t ; E_{0}\right)$ in Eq. (A1), and using standard manipulations to evaluate the double integral (see, e.g., Ref. [76]), we arrive at

$$
\operatorname{Var}(E) \approx \frac{1}{2} S\left(\omega ; E_{0}\right) \Delta t
$$

where

$$
S\left(\omega ; E_{0}\right)=\int_{-\infty}^{\infty} d t e^{-i \omega t} C\left(t ; E_{0}\right)
$$

is the power spectrum of $\dot{V}\left(z_{t}^{0}\right)$, which is equal to the Fourier transform of $C\left(t ; E_{0}\right)$ by the Wiener-Khinchin theorem [57]. The approximation in Eq. (A3) contains correction terms that are sublinear in $\Delta t$. Comparing Eq. (A3) with Eq. (14) and relabeling $E_{0}$ as $E$, we obtain Eq. (15), our final expression for $g_{2}$.

\section{Calculation of $g_{1}$}

We now derive Eq. (18), which expresses a fluctuationdissipation relation between the drift and diffusion coefficients $g_{1}$ and $g_{2}$. To do so, we first note that the constant function $\rho(z)=1$ is a stationary solution to the Liouville Eq. (2). This reflects the incompressibility of phase space volume under Hamiltonian dynamics (Liouville's theorem) [40]. Since $\rho=1$ is stationary under the dynamics in phase space, the corresponding (unnormalized) distribution in energy space should be stationary under the Fokker-Planck equation. This energy distribution, obtained by marginalizing over the constant solution $\rho=1$, is the density of states $\Sigma(E)$-see Eq. (6). Setting $\eta(E, t)=\Sigma(E)$ as a stationary solution of the Fokker-Planck Eq. (11), we have

$$
0=-\frac{\partial}{\partial E}\left[g_{1} \Sigma-\frac{1}{2} \frac{\partial}{\partial E}\left(g_{2} \Sigma\right)\right] \text {. }
$$

Thus the quantity in square brackets is constant as a function of $E$. We label this constant by $\alpha$ :

$$
\alpha \equiv g_{1} \Sigma-\frac{1}{2} \frac{\partial}{\partial E}\left(g_{2} \Sigma\right) .
$$

We now aim to show that $\alpha=0$, which then immediately implies the fluctuation-dissipation relation Eq. (18).

To proceed, we first use Eq. (A6) to eliminate $g_{1}$ from the Fokker-Planck Eq. (11), obtaining

$$
\frac{\partial \eta}{\partial t}=-\alpha \frac{\partial}{\partial E}\left(\frac{\eta}{\Sigma}\right)+\frac{1}{2} \frac{\partial}{\partial E}\left[g_{2} \Sigma \frac{\partial}{\partial E}\left(\frac{\eta}{\Sigma}\right)\right] .
$$

In the main text, in arguing that the system energy evolves diffusively, we considered trajectories with a common initial energy $E_{0}$, and we arrived at the hierarchy of timescales Eq. (12) required for the validity of the energy diffusion picture: $T \ll \tau_{C}\left(E_{0}\right) \ll \Delta t \ll \tau_{E}\left(\omega, E_{0}\right)$. Since $\tau_{E} \rightarrow \infty$ as $\omega \rightarrow \infty$, this hierarchy suggests that for a given, sufficiently large value of $\omega$, there is a range of energies over which Eq. (A7) is valid. This range can be enlarged by increasing the value of $\omega$, but there might exist no value $\omega^{*}$ such that Eq. (A7) is valid over the entire energy axis for all $\omega>\omega^{*}$. Thus let us fix the value of $\omega$ and let $[a, b]$ denote a finite interval of the energy axis, such that Eq. (A7) is valid for energies $a \leqslant E \leqslant b$. The existence of such an interval is sufficient to establish that $\alpha=0$, as we now show.

Consider an ensemble of trajectories evolving under $H(z, t)$ from an initial phase space distribution that is uniform up to a cutoff $E_{0} \in(a, b)$,

$$
\rho(z, 0)=c \theta\left(E_{0}-H_{0}(z)\right),
$$

where $\theta(\cdot)$ is the unit step function and $c^{-1}=\Omega\left(E_{0}\right)$ is the volume of phase space enclosed by the energy shell $E_{0}$ (which was assumed finite in Sec. III). The corresponding energy distribution is

$$
\begin{aligned}
\eta(E, 0) & =\int d z \delta\left(E-H_{0}\right) \rho(z, 0) \\
& =c \Sigma(E) \theta\left(E_{0}-E\right) .
\end{aligned}
$$

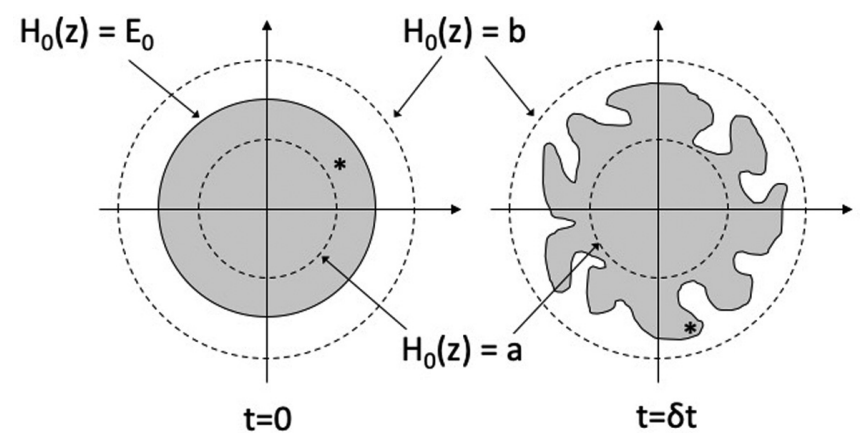

FIG. 1. Schematic depiction of phase space. On the left, the initial distribution is uniform, $\rho(z, 0)=c$ (shaded), up to a cutoff energy $E_{0}$. The figure on the right shows the distribution a short time later, after evolution under $H(z, t) ; \rho(z, \delta t)=c$ in the shaded region. Only in the annular region between the two energy shells $a$ and $b$ (dashed circles) does $\rho(z, t)$ vary with time for $t \in[0, \delta t]$. Asterisks depict initial and final conditions for a representative trajectory. 
As this ensemble of trajectories evolves in time, the value of the density $\rho$ at any $(z, t)$ is either $c$ or 0 , by Liouville's theorem. For a sufficiently short but finite interval $0 \leqslant t \leqslant$ $\delta t, \rho(z, t)$ remains constant outside the region of phase space between the two energy shells $H_{0}=a$ and $H_{0}=b$ (see Fig. 1), hence

$$
\eta(E, t)= \begin{cases}c \Sigma(E) & \text { if } E \leqslant a \\ 0 & \text { if } E \geqslant b .\end{cases}
$$

We emphasize that Eq. (A10) is exact, and a direct consequence of Liouville's theorem.
Equation (A10) implies that for $t \in[0, \delta t]$ there is no net flow of probability into or out of the energy interval $[a, b]$ :

$$
0=\frac{d}{d t} \int_{a}^{b} \eta(E, t) d E .
$$

We can use Eq. (A7), which is valid in $[a, b]$, along with Eq. (A10) to evaluate the right side of Eq. (A11), obtaining

$$
0=\left[-\alpha\left(\frac{\eta}{\Sigma}\right)+\frac{1}{2} g_{2} \Sigma \frac{\partial}{\partial E}\left(\frac{\eta}{\Sigma}\right)\right]_{a}^{b}=\alpha c,
$$

hence $\alpha=0$. By Eq. (A6), this establishes the fluctuationdissipation relation Eq. (18).
[1] B. Van der Pol and J. Van der Mark, Frequency demultiplication, Nature 120, 363 (1927).

[2] R. Chacón, Inhibition of chaos in Hamiltonian systems by periodic pulses, Phys. Rev. E 50, 750 (1994).

[3] T. M. Antonsen, R. T. Faghih, M. Girvan, E. Ott, and J. Platig, External periodic driving of large systems of globally coupled phase oscillators, Chaos 18, 037112 (2008).

[4] R. Khasseh, R. Fazio, S. Ruffo, and A. Russomanno, ManyBody Synchronization in a Classical Hamiltonian System, Phys. Rev. Lett. 123, 184301 (2019).

[5] T. Dittrich, F. Großmann, P. Jung, B. Oelschlägel, and P. Hänggi, Localization and tunneling in periodically driven bistable systems, Physica A 194, 173 (1993).

[6] T. Nag, S. Roy, A. Dutta, and D. Sen, Dynamical localization in a chain of hard core bosons under periodic driving, Phys. Rev. B 89, 165425 (2014).

[7] E. Bairey, G. Refael, and N. H. Lindner, Driving induced manybody localization, Phys. Rev. B 96, 020201(R) (2017).

[8] A. Haldar and A. Das, Dynamical many-body localization and delocalization in periodically driven closed quantum systems, Ann. Phys. 529, 1600333 (2017).

[9] E. Ott, Chaos in Dynamical Systems (Cambridge University Press, Cambridge, 2002).

[10] V. Khemani, A. Lazarides, R. Moessner, and S. L. Sondhi, Phase Structure of Driven Quantum Systems, Phys. Rev. Lett. 116, 250401 (2016).

[11] N. Y. Yao, A. C. Potter, I.-D. Potirniche, and A. Vishwanath, Discrete Time Crystals: Rigidity, Criticality, and Realizations, Phys. Rev. Lett. 118, 030401 (2017).

[12] D. V. Else, B. Bauer, and C. Nayak, Prethermal Phases of Matter Protected by Time-Translation Symmetry, Phys. Rev. X 7, 011026 (2017).

[13] J. Zhang, P. W. Hess, A. Kyprianidis, P. Becker, A. Lee, J. Smith, G. Pagano, I.-D. Potirniche, A. C. Potter, and A. Vishwanath, Observation of a discrete time crystal, Nature 543, 217 (2017).

[14] S. Choi, J. Choi, R. Landig, G. Kucsko, H. Zhou, J. Isoya, F. Jelezko, S. Onoda, H. Sumiya, and V. Khemani, Observation of discrete time-crystalline order in a disordered dipolar manybody system, Nature 543, 221 (2017).

[15] A. Russomanno, F. Iemini, M. Dalmonte, and R. Fazio, Floquet time crystal in the Lipkin-Meshkov-Glick model, Phys. Rev. B 95, 214307 (2017).
[16] N. Y. Yao, C. Nayak, L. Balents, and M. P. Zaletel, Classical discrete time crystals, Nat. Phys. 16, 438 (2020).

[17] A. Kyprianidis, F. Machado, W. Morong, P. Becker, K. S. Collins, D. V. Else, L. Feng, P. W. Hess, C. Nayak, G. Pagano, N. Y. Yao, and C. Monroe, Observation of a prethermal discrete time crystal, arXiv:2102.01695

[18] D. A. Abanin, W. De Roeck, W. W. Ho, and F. Huveneers, Effective Hamiltonians, prethermalization, and slow energy absorption in periodically driven many-body systems, Phys. Rev. B 95, 014112 (2017).

[19] A. Herrmann, Y. Murakami, M. Eckstein, and P. Werner, Floquet prethermalization in the resonantly driven Hubbard model, Europhys. Lett. 120, 57001 (2017).

[20] T. Mori, Floquet prethermalization in periodically driven classical spin systems, Phys. Rev. B 98, 104303 (2018).

[21] T. Mori, T. N. Ikeda, E. Kaminishi, and M. Ueda, Thermalization and prethermalization in isolated quantum systems: a theoretical overview, J. Phys. B 51, 112001 (2018).

[22] K. Mallayya, M. Rigol, and W. De Roeck, Prethermalization and Thermalization in Isolated Quantum Systems, Phys. Rev. X 9, 021027 (2019).

[23] O. Howell, P. Weinberg, D. Sels, A. Polkovnikov, and M. Bukov, Asymptotic Prethermalization in Periodically Driven Classical Spin Chains, Phys. Rev. Lett. 122, 010602 (2019).

[24] F. Machado, G. D. Kahanamoku-Meyer, D. V. Else, C. Nayak, and N. Y. Yao, Exponentially slow heating in short and longrange interacting Floquet systems, Phys. Rev. Research 1, 033202 (2019).

[25] A. Rajak, I. Dana, and E. G. Dalla Torre, Characterizations of prethermal states in periodically driven many-body systems with unbounded chaotic diffusion, Phys. Rev. B 100, 100302(R) (2019).

[26] F. Machado, D. V. Else, G. D. Kahanamoku-Meyer, C. Nayak, and N. Y. Yao, Long-Range Prethermal Phases of Nonequilibrium Matter, Phys. Rev. X 10, 011043 (2020).

[27] A. Rubio-Abadal, M. Ippoliti, S. Hollerith, D. Wei, J. Rui, S. L. Sondhi, V. Khemani, C. Gross, and I. Bloch, Floquet Prethermalization in a Bose-Hubbard System, Phys. Rev. X 10, 021044 (2020).

[28] P. Peng, C. Yin, X. Huang, C. Ramanathan, and P. Cappellaro, Floquet prethermalization in dipolar spin chains, Nat. Phys. (2021), doi: 10.1038/s41567-020-01120-z. 
[29] L. D'Alessio and M. Rigol, Long-Time Behavior of Isolated Periodically Driven Interacting Lattice Systems, Phys. Rev. X 4, 041048 (2014).

[30] A. Lazarides, A. Das, and R. Moessner, Equilibrium states of generic quantum systems subject to periodic driving, Phys. Rev. E 90, 012110 (2014).

[31] D. A. Abanin, W. De Roeck, and F. Huveneers, Exponentially Slow Heating in Periodically Driven Many-Body Systems, Phys. Rev. Lett. 115, 256803 (2015).

[32] J. Demers and C. Jarzynski, Universal energy diffusion in a quivering billiard, Phys. Rev. E 92, 042911 (2015).

[33] P. Ponte, A. Chandran, Z. Papić, and D. A. Abanin, Periodically driven ergodic and many-body localized quantum systems, Ann. Phys. 353, 196 (2015).

[34] J. Rehn, A. Lazarides, F. Pollmann, and R. Moessner, How periodic driving heats a disordered quantum spin chain, Phys. Rev. B 94, 020201(R) (2016).

[35] T. Mori, T. Kuwahara, and K. Saito, Rigorous Bound on Energy Absorption and Generic Relaxation in Periodically Driven Quantum Systems, Phys. Rev. Lett. 116, 120401 (2016).

[36] T. Kuwahara, T. Mori, and K. Saito, Floquet-Magnus theory and generic transient dynamics in periodically driven many-body quantum systems, Ann. Phys. 367, 96 (2016).

[37] A. Rajak, R. Citro, and E. G. Dalla Torre, Stability and prethermalization in chains of classical kicked rotors, J. Phys. A 51, 465001 (2018).

[38] S. Notarnicola, F. Iemini, D. Rossini, R. Fazio, A. Silva, and A. Russomanno, From localization to anomalous diffusion in the dynamics of coupled kicked rotors, Phys. Rev. E 97, 022202 (2018).

[39] M. C. Tran, A. Ehrenberg, A. Y. Guo, P. Titum, D. A. Abanin, and A. V. Gorshkov, Locality and heating in periodically driven, power-law-interacting systems, Phys. Rev. A 100, 052103 (2019).

[40] J. R. Dorfman, An Introduction to Chaos in Nonequilibrium Statistical Mechanics, Cambridge Lecture Notes in Physics (Cambridge University Press, Cambridge, 1999).

[41] H. Goldstein, Classical Mechanics, 2nd ed. (Addison-Wesley, Reading, MA, 1980).

[42] L. E. Reichl, A Modern Course in Statistical Physics (Arnold, London, 1980).

[43] J. A. Murdock, Perturbations: Theory and Methods (Society for Industrial and Applied Mathematics, Philadelphia, 1999).

[44] S. Rahav, I. Gilary, and S. Fishman, Effective Hamiltonians for periodically driven systems, Phys. Rev. A 68, 013820 (2003).

[45] C. W. Gardiner, Handbook of Stochastic Methods for Physics, Chemistry, and the Natural Sciences, Springer Series in Synergetics (Springer-Verlag, Berlin, 1985).

[46] E. Ott, Goodness of Ergodic Adiabatic Invariants, Phys. Rev. Lett. 42, 1628 (1979).

[47] R. Brown, E. Ott, and C. Grebogi, Ergodic Adiabatic Invariants of Chaotic Systems, Phys. Rev. Lett. 59, 1173 (1987).

[48] R. Brown, E. Ott, and C. Grebogi, The goodness of ergodic adiabatic invariants, J. Stat. Phys. 49, 511 (1987).

[49] M. Wilkinson, Dissipation by identical oscillators, J. Phys. A 23, 3603 (1990)

[50] S. Linkwitz and H. Grabert, Energy diffusion of a weakly damped and periodically driven particle in an anharmonic potential well, Phys. Rev. B 44, 11888 (1991).
[51] C. Jarzynski, Diffusion equation for energy in ergodic adiabatic ensembles, Phys. Rev. A 46, 7498 (1992).

[52] C. Jarzynski, Energy diffusion in a chaotic adiabatic billiard gas, Phys. Rev. E 48, 4340 (1993).

[53] D. Cohen, Chaos and energy spreading for time-dependent Hamiltonians, and the various regimes in the theory of quantum dissipation, Ann. Phys. 283, 175 (2000).

[54] G. Bunin, L. D'Alessio, Y. Kafri, and A Polkovnikov, Universal energy fluctuations in thermally isolated driven systems, Nat. Phys. 7, 913 (2011).

[55] S. De Bièvre and P. E. Parris, Equilibration, generalized equipartition, and diffusion in dynamical Lorentz gases, J. Stat. Phys. 142, 356 (2011).

[56] S. De Bièvre, C. Mejía-Monasterio, and P. E. Parris, Dynamical mechanisms leading to equilibration in two-component gases, Phys. Rev. E 93, 050103(R) (2016).

[57] R. Kubo, M. Toda, and N. Hashitsume, Statistical Physics II: Nonequilibrium Statistical Mechanics, Springer Series in SolidState Sciences (Springer, Berlin, 2012).

[58] If $H_{0}(z)$ has a finite range, then $\omega$ can be chosen so Eq. (11) is valid over all allowable energies. If the range of $H_{0}(z)$ is unbounded, then the extent of validity of Eq. (11) can be made arbitrarily large, though not necessarily infinite, by appropriate choice of $\omega$.

[59] W. R. Gilks, S. Richardson, and D. Spiegelhalter, Markov Chain Monte Carlo in Practice, Chapman \& Hall/CRC Interdisciplinary Statistics (Taylor \& Francis, New York, 1995).

[60] R. G. Gallager, Stochastic Processes: Theory for Applications (Cambridge University Press, Cambridge, 2013).

[61] R. N. Bracewell, The Fourier Transform and Its Applications, 2nd ed. (McGraw-Hill Kogakusha, Ltd., Tokyo, 1978).

[62] S. Rahav, I. Gilary, and S. Fishman, Time Independent Description of Rapidly Oscillating Potentials, Phys. Rev. Lett. 91 , 110404 (2003).

[63] M. Bukov, L. D'Alessio, and A. Polkovnikov, Universal high-frequency behavior of periodically driven systems: From dynamical stabilization to Floquet engineering, Adv. Phys. 64, 139 (2015).

[64] S. Higashikawa, H. Fujita, and M. Sato, Floquet engineering of classical systems, arXiv:1810.01103v2.

[65] P. V. Elyutin, Energy diffusion in strongly driven quantum chaotic systems, J. Exp. Theor. Phys. 102, 182 (2006).

[66] M. Feingold and A. Peres, Distribution of matrix elements of chaotic systems, Phys. Rev. A 34, 591 (1986).

[67] M. Wilkinson, A semiclassical sum rule for matrix elements of classically chaotic systems, J. Phys. A: Math. Gen. 20, 2415 (1987).

[68] Y. G. Sinai, Dynamical systems with elastic reflections, Russ. Math. Surv. 25, 137 (1970).

[69] L. A. Bunimovich, On the ergodic properties of nowhere dispersing billiards, Commun. Math. Phys. 65, 295 (1979).

[70] M. Wojtkowski, Principles for the design of billiards with nonvanishing Lyapunov exponents, Commun. Math. Phys. 105, 391 (1986).

[71] E. Fermi, On the origin of the cosmic radiation, Phys. Rev. 75, 1169 (1949).

[72] S. M. Ulam, On some statistical properties of dynamical systems, in Fourth Berkeley Symposium on Mathematical Statistics and Probability (University of California Press, Berkeley, California, 1961), p. 315. 
[73] A. Barnett, D. Cohen, and E. J. Heller, Rate of energy absorption for a driven chaotic cavity, J. Phys. A 34, 413 (2001).

[74] B. Batistić, Exponential Fermi acceleration in general timedependent billiards, Phys. Rev. E 90, 032909 (2014).
[75] B. V. Chirikov, A universal instability of many-dimensional oscillator systems, Phys. Rep. 52, 263 (1979)

[76] F. Reif, Fundamentals of Statistical and Thermal Physics (McGraw-Hill, Tokyo, 1965). 